\title{
Análisis comparativo 2002-2014 del riesgo volcánico en las localidades de Cherquenco y Melipeuco, Región de la Araucanía, Chile.*
}

\author{
Comparative analysis 2002-2014 of the Volcanic risk in Cherquenco and Melipeuco towns, Araucanía \\ Region, Chile.
}

\section{Javiera Millaray Tapia Valenzuela ${ }^{a}$}

Historial del artículo

Recibido:

31 de marzo de 2019.

Aceptado:

22 de julio de 2019. a Pontificia Universidad Católica de Chile, Santiago, Chile. Correo electrónico: jmtapia@uc.cl

* El presente trabajo incorpora algunos resultados del Seminario de Grado presentado para obtener el grado de Licenciado en Geografía, en la Pontificia Universidad Católica de Chile por parte de la autora, titulado "Riesgo volcánico en las localidades de Cherquenco, Melipeuco y Curacautín. Región de la Araucanía”.

\section{Palabras clave}

Amenaza, riesgo volcánico,

Volcán Llaima, vulnerabilidad

\section{Keywords}

Llaima volcano, volcanic hazards, volcanic risk, volcanic vulnerability

\section{Resumen}

Las fases subplineanas del volcán Llaima (Región de la Araucanía, Chile) representan un alto riesgo para la población de los dos asentamientos humanos más cercanos: Melipeuco y Cherquenco. De esta forma, el objetivo principal de este trabajo es calcular y analizar el riesgo volcánico en ambos pueblos, para los años 2002 y 2014. Para esto, el trabajo se dividió en tres etapas. Primero, una fase de recopilación bibliográfica, donde se buscó sentar las bases de la dinámica del volcán Llaima y su amenaza. En una segunda etapa, se determinó la vulnerabilidad de los asentamientos susceptibles a ser afectados. Finalmente, se realizó un análisis multicriterio con el objetivo de tener una primera aproximación al riesgo volcánico en el área de estudio para los años 2002 y 2014. Los resultados mostraron que las dos áreas de estudio analizadas alcanzan valores de riesgo que aumentan considerablemente en estos doce años de análisis comparativo; sobre todo en el caso de Melipeuco, donde el riesgo pasó de alto a extremo.

\section{Abstract}

The subplinian phases of the Llaima volcano (Araucanía Region, Chile) represent a high risk for the population of the two closest human settlements: Melipeuco and Cherquenco. In this way, the main objective of this work is to calculate and analyze the volcanic risk in both towns, for the years 2002 and 2014. This work was divided into three stages. First, a bibliographic compilation phase, to understand the dynamics of the Llaima volcano and its volcanic hazards. In a second stage, the vulnerability of the susceptible settlements to be affected was determined. Finally, a multicriteria analysis was carried out to have a first approximation to the volcanic risk in the study area for the years 2002 and 2014. The results showed that the two study areas analyzed have risk values that increase considerably in these twelve years of analysis comparative; especially in the case of Melipeuco, where the risk went from high to extreme. 


\section{Introducción}

Con la colonización territorial de Chile y su desarrollo demográfico, los asentamientos humanos se fueron desarrollando en áreas con características físicas poco adecuadas para su seguridad. Un ejemplo de esto son las ciudades localizadas en el borde costero o aquellas próximas a volcanes activos, entre otros (Arenas, Lagos \& Hidalgo, 2010).

En Chile, las características geográficas y distribución espacial de los asentamientos humanos aumentan los focos de vulnerabilidad y amenaza. A pesar de esto último, tanto la población como el Estado carecen de la información necesaria para tomar decisiones oportunas ante estos eventos, y prevenirlos sobre la base de la planificación territorial (Arenas et al., 2010).

Tomando en cuenta las características geológicas del territorio chileno resulta inevitable reconocer la importancia de la actividad volcánica en la modelación y estructuración del territorio chileno. Este ha sido un agente fundamental en la modificación del paisaje y en la determinación, parcial, de la dinámica de los grupos humanos que se ven influenciados por su actividad (Perry \& Lindell, 2008).

El área de estudio se encuentra en la Región de la Araucanía, a $65 \mathrm{~km}$ al este de la ciudad de Temuco. Según Naranjo y Moreno (2003) son dos los asentamientos humanos más afectados por la actividad del volcán Llaima; en primer lugar, Melipeuco, localizado a 13 kilómetros del volcán; y Cherquenco, ubicado a 22 kilómetros del mismo (Fig. 1). Dada la proximidad que tienen ambos asentamientos al volcán, es fundamental comprobar si los niveles de riesgo en el área de estudio son altos y aumentan en el tiempo. De esta forma, el objetivo principal de este trabajo es calcular y analizar el riesgo volcánico en las localidades de Cherquenco y Melipeuco a partir del análisis comparativo de la situación en el año 2002 y 2014.

El volcán Llaima se considera uno de los más con mayor volumen de los Andes del Sur, con $400 \mathrm{~km}^{3}$ y un área aproximada de $500 \mathrm{~km}^{2}$ ) (Fig. 1) (Naranjo \& Moreno, 1991). Este inició su actividad en el Pleistoceno superior, hace 250.000 años, con registros históricos existentes desde 1640. Actualmente se tienen registros de diez
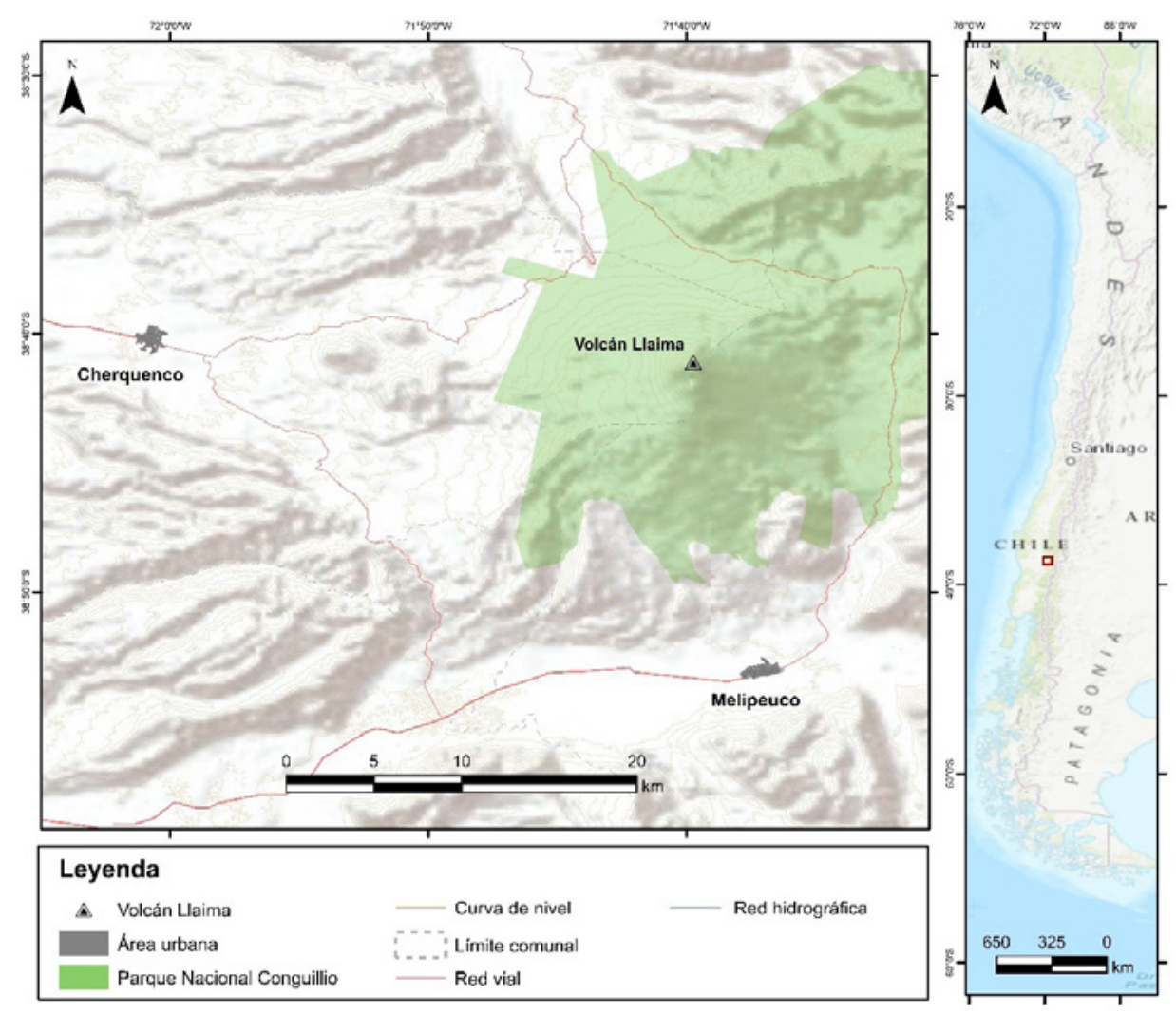

Figura 1. Esquema del proceso metodológico. Fuente: Elaboración propia. 
erupciones violentas que han generado una gran emisión de lavas y piroclastos. Según los registros históricos la actividad del volcán se ha mantenido efusiva, por lo que se establece que las erupciones del volcán Llaima son principalmente estrombolianas con fases subplineanas, y algunas de tipo hawaiano (Moreno, López \& Naranjo, 1991). Las fases subplineanas del volcán, como la sufrida en 1640, implican un elevado riesgo para la población dada su alta explosividad y generación de piroclastos.

El volcán Llaima tiene un Índice de Explosividad Volcánica (VEI) promedio de 2 (Dzierma \& Wehrmann, 2010); presentando erupciones de tipo estrombolianas, y caracterizandose por la ocurrencia de erupciones volcánicas moderadas, cuya columna de humo alcanza entre 1 y 5 $\mathrm{km}$, sin emisiones a la estratosfera. Los volúmenes de tefra generados van entre $1 \times 104 \mathrm{~m}^{3}$ a $1 \times 106 \mathrm{~m}^{3}$ (Siebert, Simkin \& Kimberly, 2010).

La información referida a la amenaza volcánica en el área de estudio ya fue levantada por SERNAGEOMIN, sin embargo, tanto la vulnerabilidad como el riesgo son desconocidos en las dos localidades más cercanas al volcán Llaima. De esta forma, determinar las áreas de riesgo volcánico es fundamental para prevenir y asegurar la población, generando la información necesaria para tomar medidas de control y mitigación pertinentes; y reducir al máximo el impacto por incertidumbre del riesgo (Doyle, McClure, Paton \& Johnston, 2014). Es así, como este estudio presenta una primera aproximación al cálculo del riesgo volcánico en la localidad de Melipeuco y Cherquenco.

\section{Conceptos Clave}

Riesgo. Según la Organización de las Naciones Unidas para la educación, Ciencia y Cultura (UNESCO) (1972) el riesgo se puede entender como la probabilidad de pérdida bajo la influencia de un evento natural. Por esta razón asume que el riesgo está determinado por la relación existente entre la vulnerabilidad y la amenaza. Posteriormente la Oficina de las Naciones Unidas para la Reducción del Riesgo de Desastres (UNISDR) postula que el concepto de riesgo se debe entender como "la combinación de la probabilidad de que se produzca un evento y sus consecuencias negativas" (UNISDR, 2009, p. 33). Este enfoque está asociado a la evaluación de riesgo, la cual es "una metodología para determinar la naturaleza y el grado de riesgo a través del análisis de posibles amenazas y la evaluación de las condiciones existentes de vulnerabilidad que conjuntamente podrían dañar potencialmente a la población, la propiedad, los servicios y los medios de sustento expuestos, al igual que el entorno de cual dependen" (UNISDR, 2009, pág. 16). Para Caselli y Vélez (2011) el riesgo natural se entiende como los procesos o fenómenos naturales que afectan a la humanidad mediante un impacto, generalmente catastrófico, sobre las vidas, instalaciones o actividades productivas. En este sentido los autores destacan el rol del riesgo volcánico en la Cordillera de los Andes, cuya actividad volcánica interactúa directamente con la actividad antrópica (Larsen, 1984). A pesar de las diferentes definiciones del concepto discutido, existe un consenso respecto su naturaleza: el riesgo se puede definir como una función de la amenaza por la vulnerabilidad. Según la ONU (UNISDR, 2009), el riesgo natural está compuesto de estos dos elementos, entiendo la amenaza como una manifestación natural cuyas características y naturaleza pueden provocar efectos negativos en la población humana (Jenkins, Spence, Fonseca, Solidum \& Wilson, 2014). Sin embargo, aún y cuando existe un acuerdo respecto a la definición del término, hay distintas interpretaciones del concepto dependiendo de la naturaleza del evento natural y el contexto sociopolítico-cultural (Shreve \& Kelman, 2014).

De esta forma, el riesgo natural se entiende como los procesos o fenómenos naturales que afectan a una sociedad mediante un impacto sobre las vidas, instalaciones o actividades productivas. Por esta razón el riesgo se compone de dos elementos: vulnerabilidad y amenaza.

Amenaza. UNISDR (2009) diseño una definición de amenaza, comprendiendo dicho término como un fenómeno que puede ocasionar la muerte, lesiones u otros impactos a la salud, igual que daños a la propiedad, servicios, sociedad y medio ambiente. Este enfoque identifica diferentes tipos de amenazas, entre los cuales destacan las de origen natural. Según esta segunda definición las amenazas naturales se pueden caracterizar según su duración, intensidad y área de influencia (Wilson, Wilson, Deligne \& Cole, 2014). La amenaza también se puede comprender desde un enfoque mucho más temporal y probabilístico. Caselli y Vélez (2011) destacan la idea de "peligrosidad volcánica", la cual es entendida como la probabilidad de que un área sea afectada por procesos o productos volcánicos cuya potencialidad destructiva se dé en un intervalo de tiempo dado. Una tercera definición del concepto es aquella que asume al peligro o amenaza como "un factor externo de riesgo, representado por la potencial ocurrencia de un suceso de origen natural, que puede manifestarse en un lugar específico, con una intensidad y duración determinadas" (Arenas et al., 2010, p. 4). Esta definición hace referencia a la potencialidad del 
evento natural sobre el espacio y tiempo, involucrando la variable espacial de manera mucho más definida.

Concluyendo, en este estudio la amenaza se comprenderá como un evento natural que tiene un potencial para causar daños en la sociedad, propiedades, servicios y medioambiente. Este evento puede manifestarse en un tiempo y lugar determinado.

Vulnerabilidad. Por otra parte, el concepto de vulnerabilidad es el segundo elemento que juega un rol fundamental en la evaluación del riesgo. Este concepto alude a las características y dinámicas de la población que puede ser afectada por una amenaza determinada (UNISDR, 2009). De esta forma, la vulnerabilidad se puede comprender como las características de una población, que los hacen susceptibles a sufrir daño ante alguna manifestación natural peligrosa (Dibben \& Chester, 1999). Es importante considerar que la vulnerabilidad de una población varia en el tiempo dependiendo de las características demográficas y socioeconómicas de una población (UNISDR, 2009). Otras definiciones abarcan no solo a la población, sino que también a la infraestructura y actividad humana que puede ser afectadas; en este sentido, se concibe como un "factor interno de riesgo de un sujeto, objeto o sistema expuesto a una amenaza, que corresponde a su disposición a ser dañado. La vulnerabilidad es multifactorial y corresponde al producto de la interacción de factores físicos, sociales, económicos y ambientales (Arenas et al., 2010, pág. 5). De esta forma, la vulnerabilidad es explicada tanto por el riesgo como por la amenaza, actuando como puente entre estos dos conceptos (Francis \& Oppenheimer, 2004). Si se analiza la vulnerabilidad desde un punto de vista más cuantitativo, esta se puede entender como la "probabilidad de que dado el evento peligroso, se genere daño; esto hace referencia a cuan propensas son las cosas a sufrir daño" (Caselli \& Vélez, 2011). Bajo este enfoque se desprende el concepto de costo. Este debe ser trabajo como la cuantificación de la perdida en términos de costo, incluyendo las reparaciones, reconstrucciones y compensaciones (UNESCO, 1972).

Finalmente, la vulnerabilidad se comprende como aquellos elementos, población o sistemas expuestos a la amenaza natural, y que dadas sus características constituyentes, son susceptibles a ser dañados (Gibb, 2018). La vulnerabilidad es un fenómeno multifactorial, compuesto por variables materiales, ambientales y humanos; lo que hace de su análisis un trabajo holístico que relaciona diferentes elementos del medio físico y humano.

\section{Materiales y métodos}

Teniendo en cuenta las características del Volcán Llaima y su proximidad a áreas pobladas, la estructura metodológica se basa en la propuesta hecha por UNESCO (1972), donde el riesgo se define como una función entre vulnerabilidad y amenaza, este estudio analizó el riesgo volcánico según la ecuación:

$\mathrm{R}=\mathrm{A} \times \mathrm{V}$

Donde:

R: Riesgo volcánico, A: Peligro volcánico y V: Vulnerabilidad La información se trabajó espacialmente utilizando Sistemas de Información Geográfica (SIG) e Imágenes Satelitales (Felpeto, Martí \& Ortíz, 2007). La información con la que se trabajó se trasladó a una grilla. Considerando la Mínima Unidad Cartografiable (UMC) cada celda tiene un valor de $50 \mathrm{~m} 2$; dado que se trabajó a nivel de manzana, y que cada manzana posee en promedio $100 \mathrm{~m} 2$.

\section{Datos empleados}

En esta primera etapa se recopiló la información ya existente. La información correspondiente a la amenaza volcánica se obtuvo a partir del "Mapa de Peligros del volcán Llaima: región de La Araucanía, escala 1:100.000" (Naranjo \& Moreno, 2003). Este mapa se encuentra en formato digital, por lo que los datos fueron ingresados a un SIG, con el fin de reclasificar la información con valores de 0 a 10 , donde 0 es una amenaza nula y 10 es una amenaza extrema. En el caso de que se trate de una asignación de carácter cualitativo, Carvacho (2011) recomienda considerar el criterio del investigador.

En cuanto a la vulnerabilidad, a diferencia del caso anterior, no existe información desarrollada de este fenómeno, por lo que se debió recopilar información necesaria para su desarrollo.

Cálculo de la vulnerabilidad y normalización

Para la determinación de la vulnerabilidad se seguirá el método propuesto por Barczak \& Grivault (2007), de tal forma que:

Donde:

$V=(a \times V h)+(b \times V a)+(c \times V m)$

$\boldsymbol{V} \boldsymbol{h}=$ Vulnerabilidad humana (número de personas susceptibles a ser dañadas).

$\boldsymbol{V} \boldsymbol{a}=$ Vulnerabilidad ambiental (área ocupada por los recursos naturales susceptibles a ser dañados). 
$\boldsymbol{V} \boldsymbol{m}=$ Vulnerabilidad material (actividades ligadas a la vida urbana y rural).

$\boldsymbol{a}, \boldsymbol{b}$ y $\boldsymbol{c}=$ Ponderación del factor.

Para el cálculo de la vulnerabilidad humana se utilizaron los datos levantados para efectos de este estudio y el censo de población y vivienda (INE, 2002). Estos fueron desarrollados con el objetivo de analizar y estimar el desarrollo y dinámicas de la vulnerabilidad humana en el área urbana, actualizando el número de habitantes por manzana al año 2014 en las localidades de Cherquenco y Melipeuco.

La vulnerabilidad ambiental se trabajó binariamente como la presencia/ausencia de los recursos naturales analizados. De esta forma, el área comprendida por los recursos fue asignada con un valor de 10 (máxima vulnerabilidad), mientras que las áreas sin puntos de interés fue asignada con puntaje 0 (vulnerabilidad nula). La información fue obtenida del Servicio Nacional de Información Territorial, Google Earth, y del "Mapa de Capacidad de Uso de Suelo" elaborado por la Corporación Nacional Forestal (CONAF). La vulnerabilidad material se relaciona con todas aquellas actividades ligadas a la vida urbana y rural. En este caso, para poder identificar las actividades del medio urbano, se consideró la infraestructura crítica dispuesta en el área de estudio: municipios u oficina de servicios públicos, hospitales, consultorios, bomberos, cárcel, estadios, colegios y carabineros. Aplicando este criterio al área de estudio, se consideraron además, los suelos con capacidad agrícola y forestal, puesto a que están directamente relacionados con las principales actividades económicas de la población. La información fue obtenida del Mapa de Capacidad de Uso de Suelo de CONAF y de la Food and Agriculture Organization (FAO) (ONU, 2004).

Cabe mencionar que la asignación de valores se llevó a cabo a partir del método propuesto por Carvacho (2011); mientras que la ponderación que se le dio a la vulnerabilidad humana, ambiental y material se determinó a partir de método de jerarquías analíticas de Saaty (1980) (Saaty, Rogers \& Pell ,1980; Papathoma-Kohle, Cristofari, Wenk \& Fuchs, 2019).

\section{Determinación del riesgo volcánico}

Una vez recopilados los datos sobre la amenaza, y generados los datos de vulnerabilidad, se aplicó la ecuación:

$\mathrm{R}=\mathrm{A} \times \mathrm{V}$
Donde:

R: Riesgo volcánico, A: Peligro volcánico y V: Vulnerabilidad

A partir de los resultados obtenidos, la información se analizó y normalizó según el método de Carvacho (2011), con valores de 0 a 10 , donde 0 representa zonas "sin riesgo" y 10 representa un "riesgo extremo" (Tabla 1).

Tabla 1

Normalización del riesgo volcánico.

\begin{tabular}{ll}
\hline Valores & Riesgo \\
\hline $0-1$ & Nulo \\
\hline $1-2$ & Extremadamente bajo \\
\hline $2-3$ & Muy bajo \\
\hline $3-4$ & Bajo \\
\hline $4-5$ & Bajo medio \\
\hline $5-6$ & Medio \\
\hline $6-7$ & Medio alto \\
\hline $7-8$ & Alto \\
\hline $8-9$ & Muy alto \\
\hline $9-10$ & Extremo \\
\hline
\end{tabular}

Fuente: Elaboración propia.

El proceso metodológico se llevó a cabo en dos escenarios: 2002 y 2014. Puesto que las variables geológicas, estructurales y medioambientales no cambiaron en el lapso de esos 12 años, la evolución del fenómeno se encuentra dado principalmente por la vulnerabilidad humana. Esta es de vital importancia, dado que la presencia constante del riesgo volcánico altera considerablemente las dinámicos socioterritoriales en la población determinando su probabilidad de sobrevivencia en el caso de eventos catastróficos (Few, Armijos \& Barclay, 2017).

Cabe destacar que, dado que el cálculo para la población el año 2014 se presenta como una estimación, los resultados se presentan como una primera aproximación al valor real.

\section{Resultados}

El riesgo natural es una situación intrínseca a la mayoría de las áreas urbanas chilenas (Arenas et al., 2010). Es por eso, que identificar las áreas de riesgo es fundamental para poder planificar los asentamientos humanos. 
Se destaca que el aumento del riesgo volcánico en las localidades de estudio está directamente relacionado con el aumento poblacional en el periodo 2002-201. Para el año 2002 Cherquenco contaba con 2.076 habitantes, mientras que el año 2014 esta cifra aumento a 2.455. En el caso de Melipeuco, para el año 2002 se tiene un total de2.243 habitantes, mientras que para el año 2014 aumenta a 3.472 habitantes. Este aumento de la población sucedió sin que ello implicase un aumento de las vías de evacuación e infraestructura urbana. Esto se fundamenta en el hecho de que la amenaza volcánica se mantiene igual, dada la escala temporal del fenómeno, por lo tanto, los cambios en el riesgo entre un año y otro radican principalmente en la vulnerabilidad. Así mismo, la vulnerabilidad material y ambiental entre un año y otro se mantiene estables, por lo que las diferencias en el riesgo se basan principalmente en el aumento poblacional de las localidades de estudio.

\section{Cherquenco}

Esta localidad presentó mayor heterogeneidad en los valores de riesgo volcánico estimados para el año 2002 y 2014 (Fig.2). Además de los cambios en la morfología del pueblo para el año 2002, toda el área urbana de Cherquenco presenta valores medios de riesgo, exceptuando algunas manzanas localizadas en el sur, cuyos valores son particularmente altos. Para el año 2014 nuevamente existe un amplio espectro en el valor del riesgo volcánico, con predominio de valores medios.

Al comparar las vulnerabilidades del año 2002 y 2014 (Fig. 3 y 4), se puede ver un cambio en la distribución de este fenómeno en el territorio. Mientras que en el año 2002 la vulnerabilidad se concentra principalmente en el sector sur de Cherquenco, zona del pueblo asociada a
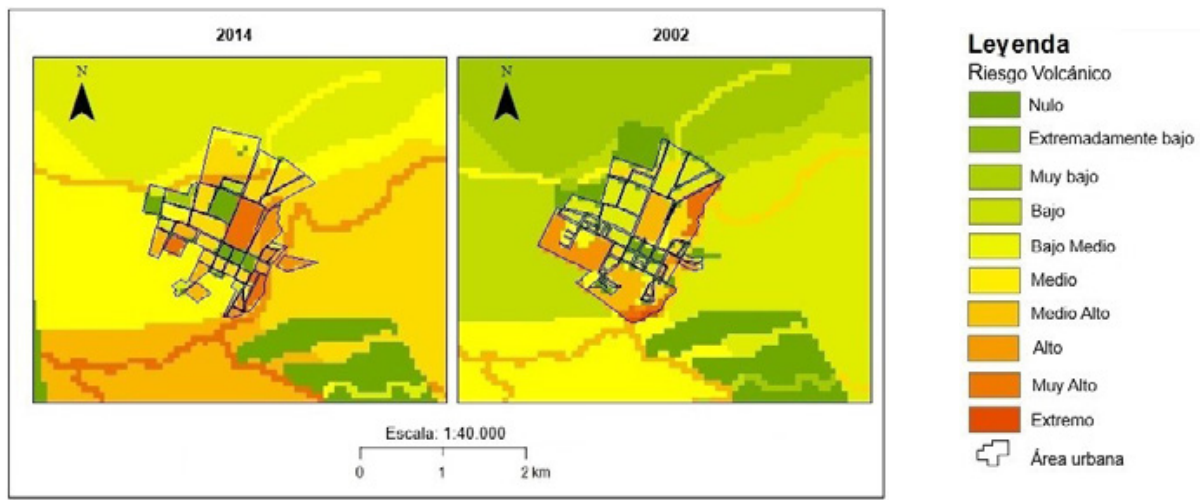

Figura 2. Mapa comparativo del riesgo volcánico en Cherquenco, para los años 2002 y 2014. Fuente: Elaboración propia.
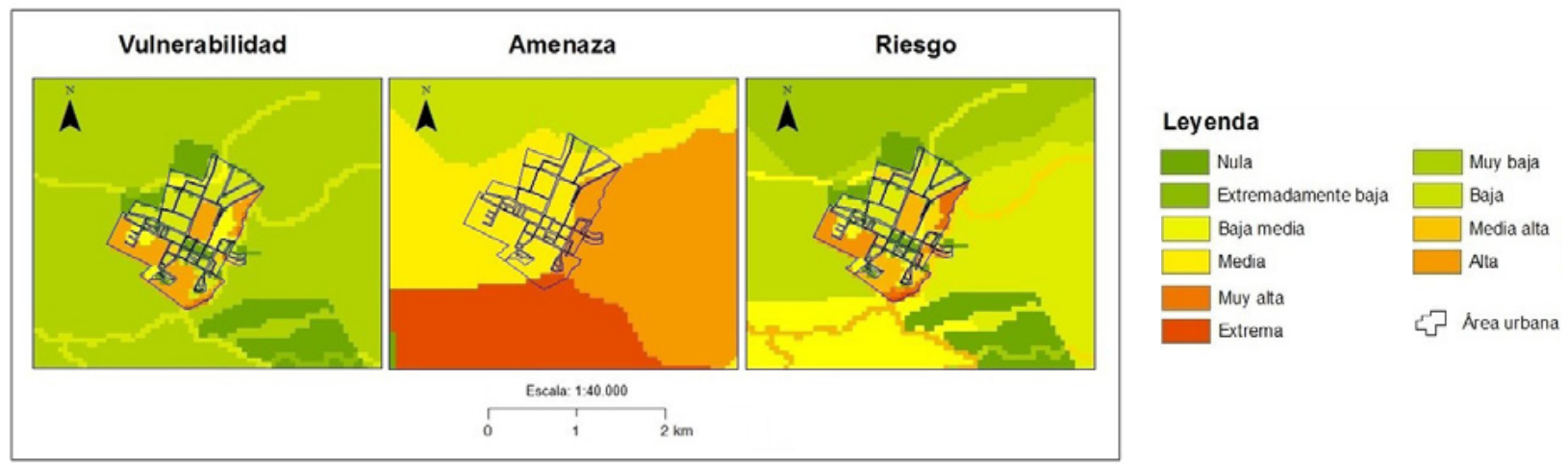

Figura 3. Mapa comparativo de la amenaza, vulnerabilidad y riesgo volcánico, Cherquenco 2002. Fuente: Elaboración propia. 


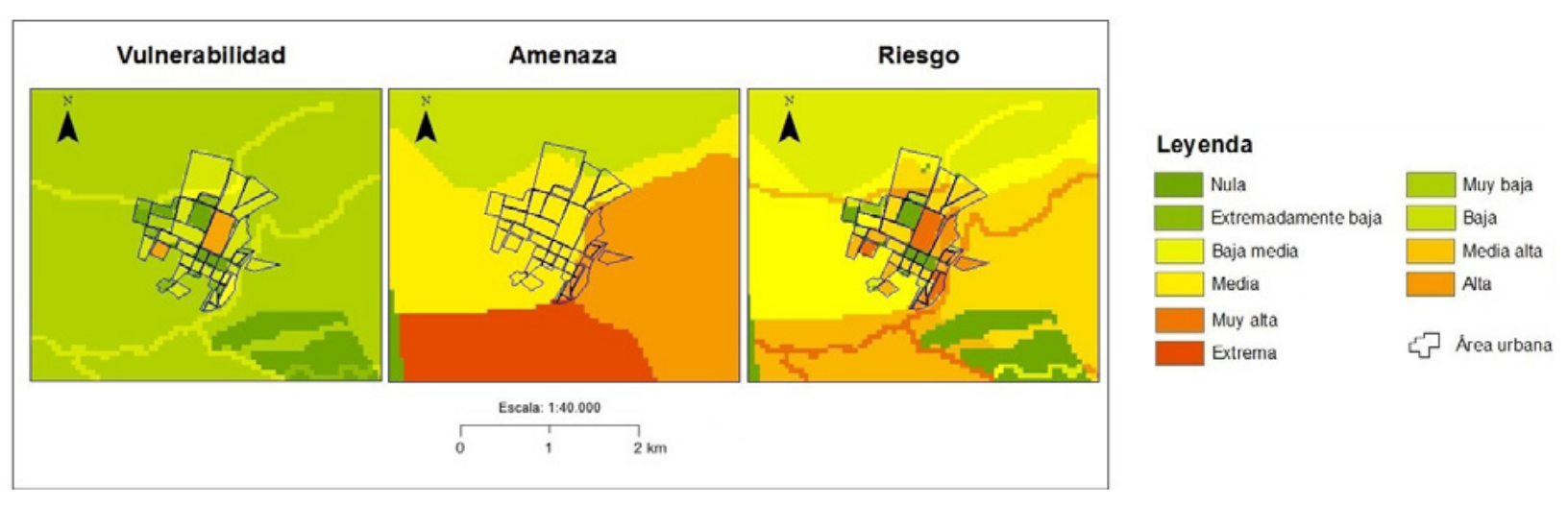

Figura 4. Mapa comparativo de la amenaza, vulnerabilidad y riesgo volcánico, Cherquenco 2014. Fuente: Elaboración propia.

actividades productivas de tipo ganadero-agrícola; en el año 2014 la vulnerabilidad se distribuye de forma más homogénea. En este punto es importante considerar que si bien, disminuye el área evaluada con mayor valor de vulnerabilidad, el valor medio de esta es mayor al encontrado para 2002. Esto se puede asociar al aumento de vivienda social en Cherquenco (Ilustre Municipalidad de Vilcún, 2011), lo que a su vez ha traído consigo un aumento de la población.

Como se observa en las figuras 3 y 4, la amenaza volcánica para ambos años es la misma; por lo que la causa del cambio en el valor de riesgo entre 2002 y 2014 reside principalmente en el aumento de la vulnerabilidad del asentamiento humano. A simple vista, el valor de vulnerabilidad para ambos años no sufre modificaciones sustanciales. Sin embargo, al ver el impacto que estos cambios generaron en el resultado de riesgo, teniendo para ambos años la misma ponderación, se puede observar la sensibilidad del fenómeno de riesgo ante la vulnerabilidad.

\section{Melipeuco}

La diferencia entre los resultados de riesgo volcánico para el año 2002 y 2014 en la localidad de Melipeuco es amplia, sobre todo, considerando que en el año 2002 existe un predominio de los valores medios y altos de riesgo volcánico, mientras que para el año 2014, estos valores aumentan a muy altos a extremos (Fig.5).

Considerando que la amenaza tiene valores extremos en toda el área de Melipeuco (Fig. 6 y 7), los valores de riesgo son considerablemente más altos en este pueblo, que en el caso de Cherquenco.

Al suroeste de Melipeuco se encuentra un área sin riesgo, dado a que, dentro de la metodología utilizada para determinar la vulnerabilidad, esa zona no cumplía con ninguna de las características analizadas e ingresadas en la determinación de la vulnerabilidad material.
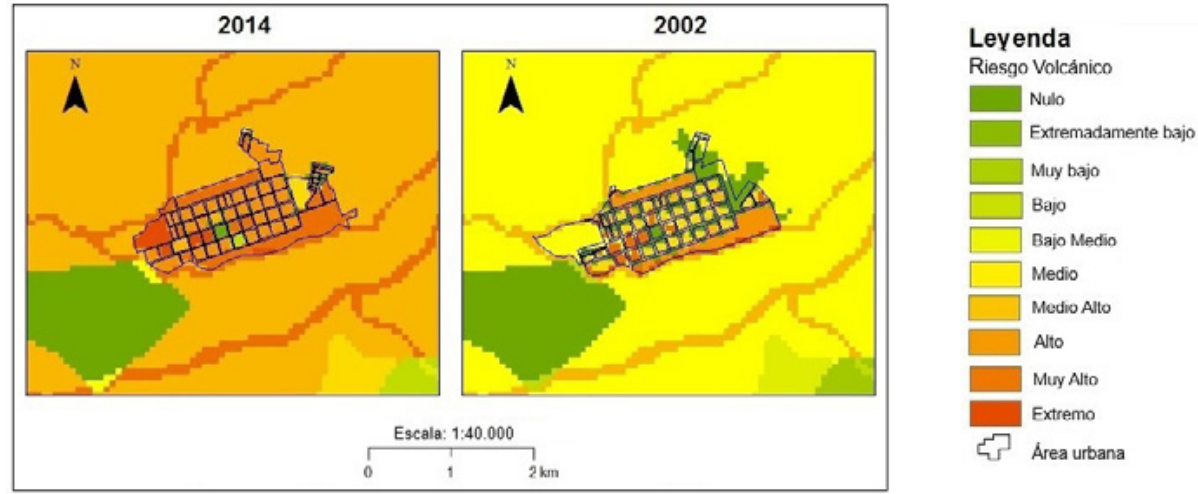

Figura 5. Mapa comparativo del riesgo volcánico en Melipeuco, para los años 2002 y 2014. Fuente: Elaboración propia. 


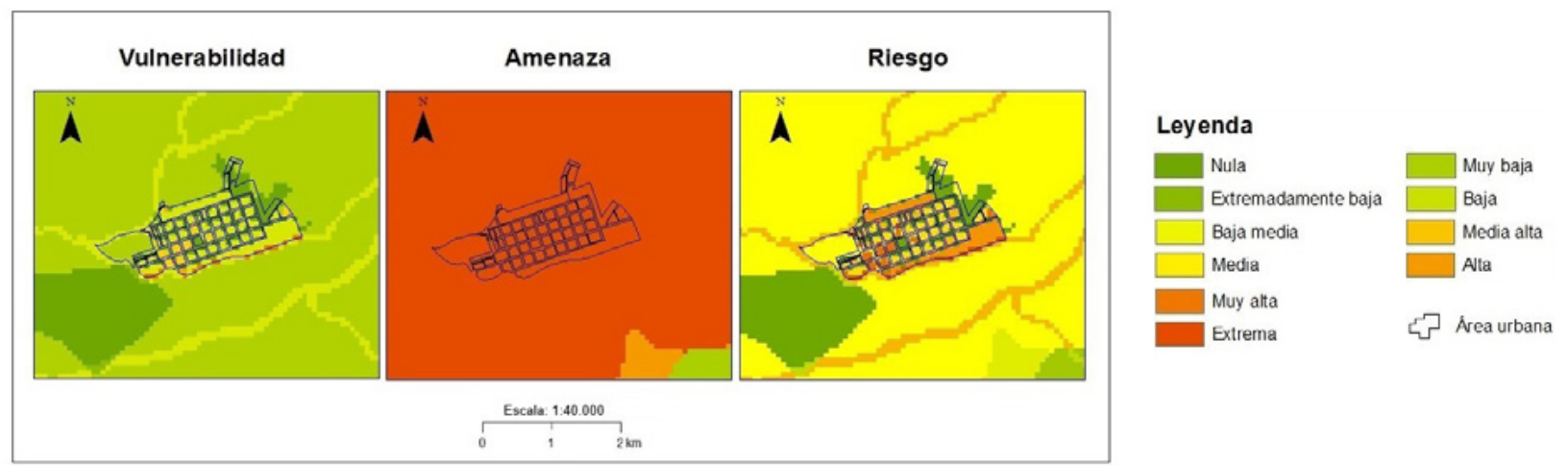

Figura 6. Mapa comparativo del riesgo volcánico y sus componentes, en Melipeuco 2002. Fuente: Elaboración propia.

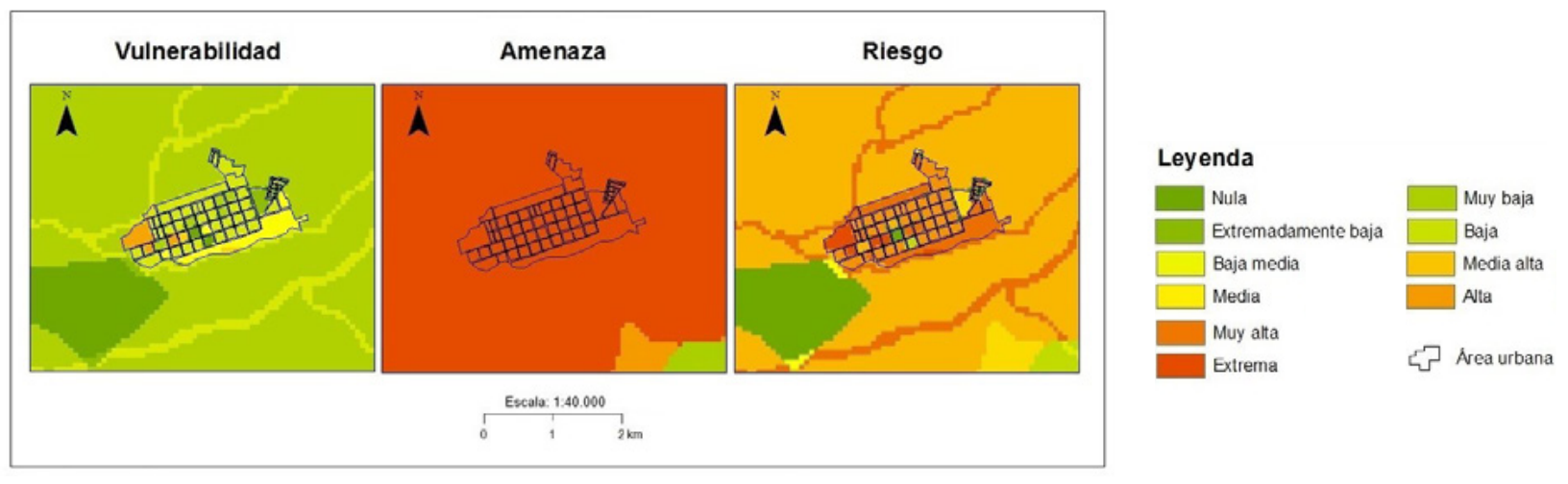

Figura 7. Mapa comparativo del riesgo volcánico y sus componentes, en Melipeuco 2014. Fuente: Elaboración propia.

$\mathrm{Al}$ igual que en el caso de Cherquenco, las principales diferencias entre el año 2002 y 2014 radican en los cambios de vulnerabilidad que se presentaron en este periodo (ej. aumento de vivienda social) (Ilustre Municipalidad de Melipeuco, 2012). Como se observa en el año 2002, la vulnerabilidad se distribuye de manera relativamente uniforme en el territorio, con una media de valores bajos; mientras que para el año 2014 los valores vuelven un poco más heterogéneos y elevan su valor medio. Como consecuencia el riesgo volcánico aumenta de valores altos para el año 2002, a extremos en el 2014.

\section{Planificación territorial y planes de emergencia}

Dentro de los instrumentos de planificación territorial del área de estudio, la información existente relacionada con el riesgo volcánico es pobre. El Plan de manejo del Parque Nacional Conguillío (CONAF, 2006) hace una breve mención sobre los efectos principales de una futura erupción, sin entrar en mayor profundidad sobre cómo afecta la actividad volcánica a las estructuras antrópicas y naturales.

Dentro de los instrumentos de planificación territorial disponibles en el área de estudio, no existe ningún tipo de aproximación al riesgo natural, a pesar de que en dicha región existen cerca de seis volcanes que poseen registros históricos de fases explosivas intensas, y de los cuales cinco cuentan con asentamientos humanos a menos de $100 \mathrm{~km}$ de distancia. Esto se encuentra directamente relacionado con la percepción del riesgo (Fevereau, Robledo \& Bull, 2018); el volcanismo, al presentarse en una escala temporal geológica, puede llegar a subestimarse si la localidad afectada se asienta en el territorio en una etapa de baja actividad eruptiva (Barberi, Davis, Isaus, Nave \& Ricci, 2008). A pesar de lo anterior, Melipeuco cuenta con un Plan de Emergencia Volcánica (Ilustre Municipalidad de Melipeuco, 2013), dada la influencia directa del volcán Llaima y el volcán Sollipulli; el primero cuenta con fases eruptivas subplineanas, y el segundo 
con fases plineanas. Además, la comuna de Melipeuco cuenta con un Plan Municipal (Ilustre Municipalidad de Melipeuco, 2015) de inversiones en reducción del riesgo de desastres orientado al desarrollo, donde se plantea la necesidad de un seminario de actualización sobre riesgo volcánico en Melipeuco.

\section{Conclusión}

El riesgo volcánico se presenta como una función entre la vulnerabilidad y la amenaza. A su vez, la vulnerabilidad se considera como un elemento determinado por factores ambientales, humanos y materiales.

Como se mencionó en los apartados anteriores, los múltiples problemas que surgieron en la gestión de la vulnerabilidad generaron que los resultados se considerarán una primera aproximación a la evaluación de riesgo volcánico.

Así mismo, tomando en cuenta que en las dos localidades de estudio se alcanzan valores de riesgo volcánico medio y alto, sobre todo en el caso de Melipeuco, donde los valores de riesgo oscilan entre alto y extremo; se recomienda tomar medidas al respecto, aplicando planes de emergencia y medidas de mitigación que sean actualizadas y oportunas. La importancia de este último punto, radica en que la construcción y fluidez en las redes institucionales del territorio son fundamentales a la hora de la toma de decisiones oportunas en situaciones de riesgo (Armijos et al., 2017).

Uno de los problemas que más destaca en la investigación fueron las dificultades presentadas con los datos de INE. En el caso de la vulnerabilidad humana, la información levantada por esta institución presentó un error de georreferenciación que generó una distorsión de los resultados calculados para el año 2002. Del mismo modo, la ausencia de información del CENSO 2012, generó la necesidad de estimar los datos, sin poder contar con un valor concreto del número de personas por manzana para el año 2014.

Otro problema importante encontrado fue que la información de amenaza volcánica solo considera la amenaza volcánica generada por coladas de lava y lahares, lo cual generó una subestimación del peligro volcánico, y por ende una subestimación en los resultados de riesgo volcánico. En este sentido, cabe destacar que la evaluación del riesgo natural es un campo multidisciplinario, donde se necesitan diferentes enfoques para poder realizar un análisis completo que responda a la realidad de manera efectiva. En el caso de este estudio, sólo se trabaja la evaluación de riesgo volcánico desde un enfoque geográfico, por lo que se resalta la necesidad, para futuras trabajos, de poder poner en práctica distintos enfoques, técnicas y conocimiento. A pesar de lo anterior, el área de estudio se considera un área sumamente sensible, que necesita la consolidación de políticas y planes de emergencia coherentes con la realidad de su medio natural (Jones, Oven, Manyena \& Aryal., 2014). Un ejemplo claro de la poca claridad que se tiene del contexto territorial, se expresa en el aumento de vivienda social en el área de estudio, y su relación con el aumento del riesgo volcánico. En este sentido, la generación de nueva información consistente y actualizada es fundamental para poder entender las dinámicas medio ambientales y humanas que surgen en el territorio. Los instrumentos de planificación territorial presentes no logran sostener la magnitud del fenómeno, y por lo tanto, generar medidas de control efectivas. A esto se le debe agregar la falta de dinamismo en la vulnerabilidad material, donde las estructuras antrópicas no presentan grandes cambios entre un periodo y otro, por lo que el acceso a servicios básicos sigue siendo el mismo a pesar del aumento de la población, generando una mayor presión y demanda. Por otra parte, la educación y toma de consciencia sobre la amenaza volcánica es fundamental para mejorar la reacción ante una futura erupción volcánica (Barberi, Davis, Isaus, Nave \& Ricci, 2013); por lo que en futuras investigaciones, conocer la percepción de riesgo por parte de la población, así como comprender la interpretación de las vivencia del riesgo por parte de la población, es clave para mejorar los tiempos de reacción ante un evento de riesgo (Eiser et al., 2012).

Es así como este estudio, al ser una primera aproximación de la evaluación de riesgo volcánico, abre las puertas a nuevas investigaciones que complementen y profundicen en el análisis de la amenaza y vulnerabilidad del área de estudio.

\section{Referencias}

Armijos, M., Phillips, J., Wilkinson, E., Barclay, J., Hicks, A., Palacios, P., Mothes, P., Stone, J. (2017). Adapting to changes in volcanic behaviour: Formal and informal interactions for enhanced risk management at Tungurahua Volcano, Ecuador. Global Environmental Change, 45, 217-226. https:// doi.org/10.1016/j.gloenvcha.2017.06.002

Arenas, F., Lagos, M., \& Hidalgo, R. (2010). Los riesgos naturales en la planificación territorial. Centro de politicos públicas UC. Santiago, Chile 
Barberi, F., Davis, M.S., Isaia, R., Nave, R., Ricci, T. (2008) Volcanic risk perception in the Vesuvius population. Journal of Volcanology and Geothermal Research, 172(3-4), 244 - 258. https://doi. org/10.1016/j.jvolgeores.2007.12.011

Barberi, F., Davis, M.S., Isaia, R., Nave, R., Ricci, T. (2013). Volcanic risk perception in the Campi Flegrei area. Journal of Volcanology and Geothermal Research, 254, 118 - 130. https://doi.org/10.1016/j. jvolgeores.2013.01.002

Barczak, A., \&Grivault, C. (2007). Geographical Information System for the assessment of vulnerability to urban surface runoff. NOVATECH. GRAIE, Lyon, France

Carvacho, L. (2011). ASIGCON, propuesta de un método para la comparación de unidades espaciales utilizando variables normalizadas sobre una base conceptual. Anales de la Sociedad Chilena de Ciencias Geográficas, 448-455.

Caselli, A., \& Vélez, M. L. (2011). Riesgo volcánico. Relatorio del XVIII congreso geológico argentino. Neuquén: Grupo de Estudio y Seguimiento de Volcanes Activos, Universidad de Buenos Aires.

CONAF (2006). Plan de Manejo Parque Nacional Conguillío. Documento de Trabajo $N^{\circ} 405$.

Dibben, C., Chester, D. (1999). Human vulnerability in volcanic environments: the case of Furnas, São Miguel, Azores. Journal of Volcanology and Geothermal Research, 92 (1-2), 133-150. https://doi. org/10.1016/S0377-0273 (99)00072-4

Doyle, E., McClure, J., Paton, D., Johnston, D. (2014). Uncertainty and decision making: Volcanic crisis scenarios. International Journal of Disaster Risk Reduction, 10 (A), 75-101. https://doi.org/10.1016/j. ijdrr.2014.07.006

Dzierma, Y., \& Wehrmann, H. (2010). Eruption time series statistically examined: Probabilities of future eruptions at Villarrica and Llaima Volcanoes, Southern Volcanic Zone, Chile. Journal of Volcanology and Geothermal Research. Elsevier, 82-92.

Eiser, J., Bostrom, A., Burton, I., Johnston, D., McClure, J., Paton, D., Van der Pligt, J., White, M. (2012). Risk interpretation and action: A conceptual framework for responses to natural hazards. International Journal of Disaster Risk Reduction, 1, 5-16. https://doi. org/10.1016/j.ijdrr.2012.05.002

Felpeto, A., Martí, J., Ortiz, R. (2007). Automatic GIS-based system for volcanic hazard assessment. . Journal of Volcanology and Geothermal Research, 166 (2), 106-116. https://doi.org/10.1016/j. jvolgeores.2007.07.008

Fevereau, M., Robledo, L., Bull, M. (2018). Analysis of risk assessment factors of individuals in volcanic hazards: Review of the last decade. Journal of Volcanology and Geothermal Research, 357, 254-260. https://doi.org/10.1016/j.jvolgeores.2018.05.009

Few, R., Armijos, M., Barclay, J. (2017). Living with Volcan Tungurahua: The dynamics of vulnerability during prolonged volcanic activity. Geoforum, 80, 7281. https://doi.org/10.1016/j.geoforum.2017.01.006

Francis, P., \& Oppenheimer, C. (2004). Volcanoes. New York: Oxford University Press.

Gibb, C. (2018). A critical analysis of vulnerability. International Journal of Disaster Risk Reduction, 28, 327-334. https://doi.org/10.1016/j.ijdrr.2017.11.007.

Ilustre Municipalidad de Melipeuco. (2012). Plan de Desarrollo Comunal. Comuna de Melipeuco 20122016

Ilustre Municipalidad de Melipeuco. (2013). Plan de Emergencia volcánica 2013.

Ilustre Municipalidad de Melipeuco. (2015). Plan municipal de inversiones en reducción del riesgo de desastres orientado al desarrollo.

Ilustre Municipalidad de Vilcún. (2011). PLADECO Vilcún 2011-2014.

INE. (2002). Población, número de viviendas, superficie, densidad de viviendas y habitantes por vivienda. Región de La Araucanía. Censo 2002.

Jenkins, S., Spence, R., Fonseca, J., Solidum, R., Wilson, T. (2014). Volcanic risk assessment: Quantifying physical vulnerability in the built environment. Journal of Volcanology and Geothermal Research, 105-120. https://doi.org/10.1016/j.jvolgeores.2014.03.002 
Jones, S., Oven, K., Manyena, B., Aryal, K. (2014). Governance struggles and policy processes in disaster risk reduction: A case study from Nepal. Geoforun, 54, 78-90. https://doi.org/10.1016/j. geoforum.2014.07.011

Larsen, G., (1984). Recent volcanic history of the Veidivötn fissure swarm, southern Iceland - an approach to volcanic risk assessment. Journal of Volcanology and Geothermal Research, 22 (1-2), 3358. https://doi.org/10.1016/0377-0273 (84)90034-9

Moreno, H., López, L., \& Naranjo, J. (1991). Volcán Llaima: geología, petrología y evaluación de riesgo volcánico. Proyecto 1048 FONDECYT.

Naranjo, J., \& Moreno, H. (1991). Actividad explosiva postglacial en el volcán Llaima, Andes del Sur. Santiago: Servicio Nacional de Geología y Minería.

Naranjo, J., \& Moreno, H. (2003). Mapa de riesgos del Volcán Llaima, Región de la Araucanía. Chile: Servicio Nacional de Geología y Minería.

ONU. (2004). FAO. Recuperado el 2014, de Food and Agriculture Organization: http://www.fao.org/

Papathoma-Kohle, M., Cristofari, G., Wenk, M., Fuchs, S. (2019). The importance of indicator weights for vulnerability indices and implications for decision making in disaster management. International Journal of Disaster Risk Reduction. https://doi.org/10.1016/j. ijdrr.2019.101103

Perry, R., Lindell, M. (2008). Volcanic risk perception and adjustment in a multi-hazard environment. Journal of Volcanology and Geothermal Research, 172 (3-4), 170 - 178. https://doi.org/10.1016/j. jvolgeores.2007.12.006

Saaty, T.L. (1980), The Analytic Hierarcy Process. McGraw-Hill, New York.

Saaty, Rogers y Pell (1980), Portfolio selection through hierarchies. In Journal of Porfolio Management, 6/3, $16-21$.

Shreve, C.M., Kelman, I. (2014). Does mitigation save? Reviewing cost-benefit analyses of disaster risk reduction. International Journal of Disaster Risk Reduction, 10 (A), 213-235. https://doi.org/10.1016/j. ijdrr.2014.08.004
Siebert, L., Simkin, T., \& Kimberly, P. (2010). Volcanoes of the World (third edition.). Smithsonian Institute: University of California Press.

UNESCO. (1972). Consultative meeting of experts on the statistical study of natural hazards and their consequences. Paris.

UNISDR (2009). Estrategia Internacional para la Reducción de Desastres. Terminología sobre Reducción de riesgos de desastres. Ginebra, Suiza.

Wilson, G., Wilson, T., Deligne, N., Cole, J. (2014). Volcanic hazard impacts to critical infrastructure: A review. Journal of Volcanology and Geothermal Research, 286, 148-182. https://doi.org/10.1016/j. jvolgeores.2014.08.030 